\title{
Changes in invasive pneumococcal disease serotypes in a regional area of Australia following three years of 7vPCV introduction
}

\author{
Tove Fitzgerald, a Peter D Massey a and Fakhrul Islam ${ }^{a}$ \\ Correspondence to Tove Fitzgerald (e-mail: Tovelysa.Fitzgerald@hnehealth.nsw.gov.au)
}

Background: Invasive pneumococcal disease (IPD) is a serious bacterial disease. Vaccination can prevent disease for many of the current serotypes. The aim of this investigation was to describe the notification rates of IPD in a regional area of Australia, explore changes in rates since the introduction of the population vaccine programmes in 2005 and to describe changes in the distribution of serotypes in relation to the available vaccines after three years.

Methods: Annualized IPD notification rates were calculated for residents of a regional area in northern New South Wales. Rates were analysed according to serotypes covered by available vaccines. Changes in serotypes were compared for the periods 2002-2004 and 2008-2010.

Results: The annualized notification rate of IPD in all ages for the period 2002-2004 was 13.7 per 100000 population, and 8.3 per 100000 population for the period 2008-2010 (rate ratio [RR], 0.61, confidence interval [CI]: 0.51-0.72). The largest decline was observed in 7-valent pneumococcal conjugate vaccine (7vPCV) types across all age groups $(\mathrm{RR}, 0.17, \mathrm{Cl}: 0.12-0.24)$ and in the zero to four year age group (RR, $0.03, \mathrm{Cl}: 0.01-0.11)$. The six serotypes included in the new 13-valent pneumococcal conjugate vaccine, but not in the 7vPCV, accounted for $40.6 \%$ of IPD cases in the zero to four year age group during the period of 2008-2010.

Discussion: The introduction of 7vPCV significantly reduced the overall notification rate of IPD caused by the serotypes contained in this vaccine. This decline in IPD rates in children can be directly attributed to the use of 7vPCV, and in adults it is most likely an indirect effect of the 7vPCV programme in children.

nvasive pneumococcal disease (IPD) is a serious bacterial infection caused by the bacteria Streptococcus pneumoniae and can result in bacteraemia, meningitis and pneumonia. Higher rates of the disease are seen in children less than two years of age, in older adults and in people who are immunosuppressed or have a chronic illness. ${ }^{1}$

There are 90 known capsular antigenic types of Streptococcus pneumoniae. Some serotypes are carried in the upper respiratory tract and others are more associated with the invasive disease. Prior to the introduction of pneumococcal vaccines into the Australian immunization schedule, $83 \%-85 \%$ of IPD cases in children aged less than 14 years and $69 \%$ of IPD cases in adults aged more than 65 years in the state of New South Wales (NSW) could be attributed to the seven serotypes found in the 7-valent pneumococcal conjugate vaccine (7vPCV) (Box 1). ${ }^{2}$

\section{Box 1. Serotypes contained in 7vPCV, 13vPCV and 23PPV, Australia

\begin{tabular}{cl}
\hline & \multicolumn{1}{c}{ Serotypes } \\
\hline 23vPPV & 12345 6B 7F 8 9N 9V 10A 11A 12F 14 \\
& 15B 17F 18C 19A 19F 20 22F 23F 33F \\
7vPCV & 46B 9V 14 18C 19F 23F \\
13vPCV & 13456A 6B 7F 9V 14 18C 19A 19F 23F \\
\hline
\end{tabular}

A vaccine programme has been in place in Australia for pneumococcal disease since 1999 when the 23-valent polysaccharide pneumococcal vaccine (23vPPV) was introduced for Aboriginal and Torres Strait Islander adults aged 50 years and over (Box 2). ${ }^{1}$ This was extended to non-Aboriginal people from 2005 for those aged 65 years and over. The vaccine is also recommended for people less than 65 years of age who have conditions that predispose them to IPD. ${ }^{1}$

\footnotetext{
a Hunter New England Population Health, Newcastle, Australia. 


\section{Box 2. Summary of funded Pneumococcal Immunization Programme, Australia}

\begin{tabular}{cl}
\hline Year & \multicolumn{1}{c}{ Vaccine } \\
\hline 1999 & Pneumovax $23^{\text {TM }}$ introduced for Aboriginal and Torres Strait Islander adults $>50$ years of age \\
2001 & Prevenar $7^{\text {TM }}$ introduced for Aboriginal and Torres Strait Islander children in Central Australia only \\
2003 & Prevenar $7^{\text {TM }}$ introduced for all children less than five years of age with medical risks \\
2005 & $\begin{array}{l}\text { Prevenar } 7^{\text {TM }} \text { introduced for all children at two, four and six months of age with catch-up for children } \\
\text { born between } 1 \text { January } 2003 \text { to } 31 \text { December } 2004\end{array}$ \\
& Pneumovax 23 $23^{\text {TM }}$ introduced for all adults $>65$ years of age \\
2011 & Prevenar $13^{\text {TM }}$ introduced for all children at two, four and six months of age \\
\hline
\end{tabular}

The 7vPCV, which comprises seven of the serotypes found in the 23vPPV, was introduced into the Australian immunization schedule for medically at-risk and Aboriginal and Torres Strait Islander children in $2001 .{ }^{1}$ In 2005, the schedule for 7vPCV was extended to include all children up to two years of age, including a catch-up programme. A new 13-valent vaccine (13vPCV) was introduced into the Australian immunization schedule in June 2011, comprising an additional five serotypes also found in 23vPPV, as well as one unique serotype (Box 1).

IPD has been notifiable by laboratories in NSW, Australia, since December 2000 under the NSW Public Health Act 2010. Notification and surveillance data are entered into the NSW Notifiable Conditions Information Management System by Public Health Units and include data on serotype when testing is completed.

There have been some reports of the impact of the pneumococcal vaccine since its introduction into the Australian immunization schedule and the potential benefits that may be offered by the 13vPVC. A national study of IPD in children less than two years old reported a $74 \%$ decline in incidence of IPD post introduction of the pneumococcal vaccine in 2005 with a $97 \%$ decline in cases caused by 7vPCV types. ${ }^{3}$ At that time, 50\% of IPD cases were caused by serotypes contained in 13vPCV.

In the regional area of North Queensland, IPD rates have declined by $34 \%$ across all age groups after introduction of 7vPVC. Declines in the number of IPD cases caused by serotypes contained in 7vPCV across age groups not targeted for vaccination demonstrated that 7vPCV had a population effect. ${ }^{4}$ The authors of this study estimated that a further $64 \%$ of IPD cases could be prevented by the introduction of 13vPCV through direct and indirect effects. Different areas across Australia likely have different IPD epidemiology.

The Hunter New England Local Health District has a population of approximately 840000 and consists of both rural and urban landscapes over $130000 \mathrm{~km}^{2}$. Approximately $22 \%$ of the NSW Aboriginal and Torres Strait Islander population reside in this area. The proportion of the population aged 65 years and over is approximately $17 \%$ and is projected to increase to $25 \%$ by $2030 .^{5}$

The aim of this investigation was to describe the notification rates of IPD before and after the introduction of the vaccine programmes, in a regional area in the state of New South Wales, Australia. An additional aim was to explore changes in the distribution of serotypes of the notified cases in relation to the available vaccines.

\section{METHODS}

IPD notification data for the period 2002-2010 in the regional area of Hunter New England (HNE) in northern NSW were sourced from the Health Outcomes Information and Statistical Toolkit, NSW Ministry of Health. Analysis was performed using Statistical Analysis Software (SAS) version 9.2. IPD notification rates were calculated using mid-year Estimated Residential Population figures from the Australian Bureau of Statistics from 2002 to 2010.

Annualized notification rates by serotype were calculated for two comparison periods, 2002-2004 and 2008-2010. These correspond with the period before the introduction of the free pneumococcal vaccine to 
Figure 1. IPD notifications by age group, Hunter New England, NSW, 2002 to 2010

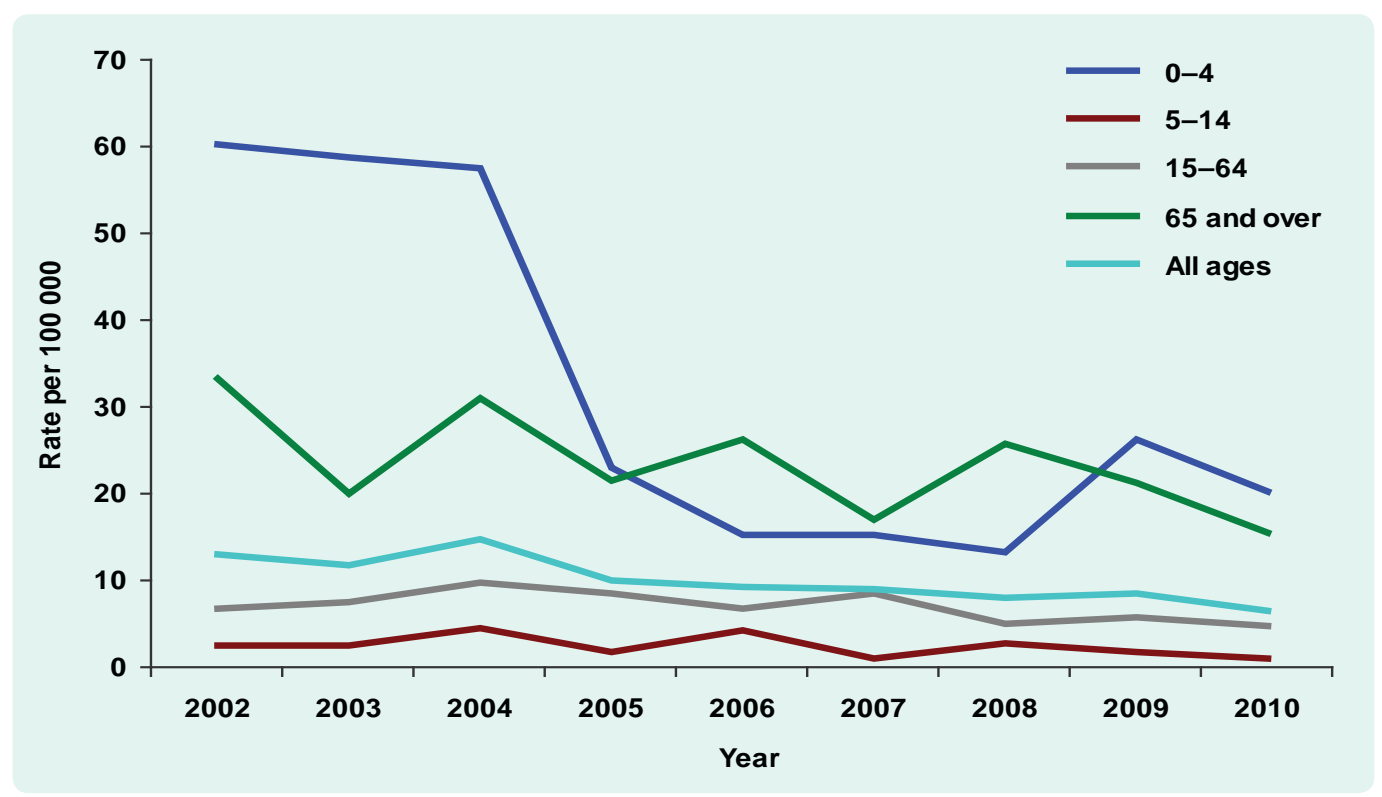

the Australian immunization schedule in 2005 and the period after. Rate ratios (RR) were calculated comparing the notification rates of the period 2008-2010 to the period of 2002-2004 notification rate baseline with their $95 \%$ confidence interval $(\mathrm{Cl})$.

Serotypes were grouped into 7vPCV, 23vPPV, 23PPV-only (16 serotypes) and non-vaccine types. Serotypes notcontained in any pneumococcal vaccinewere termed non-vaccine types. The additional six serotypes found in $13 v P C V$ are also described (referred to as $6 v[13 v-7 v])$.

This project was deemed a quality improvement exercise by the HNE Human Research Ethics Committee and did not require ethics approval.

\section{RESULTS}

The total number of laboratory-confirmed IPD cases for the period 2002-2004 was 339, and for the period 2008-2010 there were 218 notified cases. The proportion of untyped specimens was 16\% (54/339 between 2002 and 2004 and 6\% $(13 / 218)$ in the period 2008-2010. The annualized notification rate of IPD for all ages for the period 2002-2004 was 13.7 per 100000 population and 8.3 per 100000 population for the period 2008-2010 - a RR of 0.61 (95\% Cl:0.51-0.72). Across the age groups the notification rate of IPD in the zero to four years age group has shown the largest reduction (Figure 1).
The overall notification rate in males was greater than females at 16.6 per 100000 and 9.3 per 100000 , respectively, in the period 2002-2004 and 10.0 per 100000 in males and 6.2 per 100000 in females in the period 2008-2010. The largest decline was observed in notifications of IPD cases caused by 7vPCV serotypes with a rate ratio of 0.17 (95\% Cl: $0.12-0.24)$. A decline was also observed in the notification rate of IPD caused by 23PPV types (RR, 0.55, 95\% Cl: 0.45-0.67).

An increase was observed in the notification rate of IPD cases with 23PPV only types (RR, 1.99, $95 \% \mathrm{Cl}: 1.43-2.73)$. The notification rate of IPD with non-vaccine types also increased with a RR of $3.18(95 \% \mathrm{Cl}: 1.84-5.49)$. An increase was noted in IPD cases caused by $6 \mathrm{v}(13 \mathrm{v}-7 \mathrm{v})$ types (RR, 2.43, 95\% Cl: $1.61-3.66)$. In fact, $40.6 \%$ of cases in the zero to four years age group were caused by $6 v(13 v-7 v)$ types (Table 1$)$.

Significant increases were recorded for 23PPV only, non-7vPCV and $6 \mathrm{~V}(13 \mathrm{v}-7 \mathrm{v})$ types in the $0-4$, 15-64 and 65+ years age groups. Additionally, there was also a significant increase for non-vaccine types in the 15-64 and 65+ years age groups. Significant decreases were seen in the total, 23PPV and 7vPCV types for these age groups. There was no significant difference between the notification rates of IPD for 5 to 14-year-olds between the periods 2002-2004 and 2008-2010 (Table 1). 


\section{Table 1. Changes in IPD notification rates by age group and serotype, Hunter New England, 2002-2004 and 2008-2010}

\begin{tabular}{|c|c|c|c|c|c|c|c|c|}
\hline \multirow[b]{2}{*}{ Vaccine } & \multicolumn{3}{|c|}{ 2002-2004 } & \multicolumn{3}{|c|}{ 2008-2010 } & \multirow[b]{2}{*}{ Rate ratio } & \multirow[b]{2}{*}{$95 \% \mathrm{Cl}$} \\
\hline & Number & $\%$ & $\begin{array}{l}\text { Rate per } \\
100000\end{array}$ & Number & $\%$ & $\begin{array}{c}\text { Rate per } \\
100000\end{array}$ & & \\
\hline \multicolumn{9}{|l|}{ All ages } \\
\hline Total & 339 & 100.0 & 13.7 & 218 & 100.0 & 8.3 & 0.61 & $0.51-0.72$ \\
\hline 23vPPV & 271 & 79.9 & 10.9 & 157 & 72.0 & 6.0 & 0.55 & $0.45-0.67$ \\
\hline $7 \mathrm{vPCV}$ & 215 & 63.4 & 8.7 & 39 & 17.9 & 1.5 & 0.17 & $0.12-0.24$ \\
\hline 23vPPV only & 56 & 16.5 & 2.3 & 118 & 54.1 & 4.5 & 1.99 & $1.43-2.73$ \\
\hline Non-7vPCV & 70 & 20.6 & 2.8 & 165 & 75.7 & 6.3 & 2.23 & $1.69-2.95$ \\
\hline Non-vaccine & 14 & 4.1 & 0.6 & 47 & 21.6 & 1.8 & 3.18 & $1.84-5.49$ \\
\hline $6 v(13 v-7 v)$ & 32 & 9.4 & 1.3 & 82 & 37.6 & 3.1 & 2.43 & $1.61-3.66$ \\
\hline \multicolumn{9}{|l|}{$0-4$ years old } \\
\hline Total & 92 & 100.0 & 58.2 & 32 & 100.0 & 20.0 & 0.34 & $0.23-0.51$ \\
\hline 23vPPV & 74 & 80.4 & 46.8 & 23 & 71.9 & 14.3 & 0.31 & $0.19-0.49$ \\
\hline $7 \mathrm{vPCV}$ & 72 & 78.3 & 45.6 & 2 & 6.3 & 1.2 & 0.03 & $0.01-0.11$ \\
\hline 23vPPV only & 2 & 2.2 & 1.3 & 21 & 65.6 & 13.1 & 10.35 & $2.42-44.12$ \\
\hline Non-7vPCV & 5 & 5.4 & 3.2 & 29 & 90.6 & 18.1 & 5.72 & $2.21-14.76$ \\
\hline Non-vaccine & 3 & 3.3 & 1.9 & 8 & 25.0 & 5.0 & 2.63 & $0.70-9.90$ \\
\hline $6 v(13 v-7 v)$ & 2 & 2.2 & 1.3 & 13 & 40.6 & 8.1 & 6.40 & $1.44-28.38$ \\
\hline \multicolumn{9}{|c|}{ 5-14 years old } \\
\hline Total & 11 & 100.0 & 3.1 & 6 & 100.0 & 1.8 & 0.56 & $0.21-1.52$ \\
\hline 23vPPV & 10 & 90.9 & 2.8 & 3 & 50.0 & 0.9 & 0.31 & $0.08-1.12$ \\
\hline $7 \mathrm{vPCV}$ & 8 & 72.7 & 2.3 & 2 & 33.3 & 0.6 & 0.26 & $0.05-1.21$ \\
\hline 23vPPV only & 2 & 18.2 & 0.6 & 1 & 16.7 & 0.3 & 0.51 & $0.05-5.68$ \\
\hline Non-7vPCV & 2 & 18.2 & 0.6 & 4 & 66.7 & 1.2 & 2.06 & $0.38-11.24$ \\
\hline Non-vaccine & 0 & 0.0 & 0.0 & 3 & 50.0 & 0.9 & - & - \\
\hline $6 v(13 v-7 v)$ & 2 & 18.2 & 0.6 & 1 & 16.7 & 0.3 & 0.51 & $0.05-5.68$ \\
\hline \multicolumn{9}{|c|}{$15-64$ years old } \\
\hline Total & 131 & 100.0 & 8.2 & 91 & 100.0 & 5.4 & 0.65 & $0.50-0.85$ \\
\hline 23vPPV & 107 & 81.7 & 6.7 & 73 & 80.2 & 4.3 & 0.64 & $0.48-0.86$ \\
\hline $7 \mathrm{vPCV}$ & 75 & 57.3 & 4.7 & 20 & 22.0 & 1.2 & 0.25 & $0.15-0.41$ \\
\hline 23vPPV only & 32 & 24.4 & 2.0 & 53 & 58.2 & 3.1 & 1.56 & $1.00-2.42$ \\
\hline Non-7vPCV & 35 & 26.7 & 2.2 & 65 & 71.4 & 3.8 & 1.75 & $1.16-2.64$ \\
\hline Non-vaccine & 3 & 2.3 & 0.2 & 12 & 13.2 & 0.7 & 3.77 & $1.06-13.35$ \\
\hline $6 v(13 v-7 v)$ & 16 & 12.2 & 1.0 & 35 & 38.5 & 2.1 & 2.06 & $1.14-3.72$ \\
\hline \multicolumn{9}{|c|}{65 years old and above } \\
\hline Total & 105 & 100.0 & 28.0 & 89 & 100.0 & 20.9 & 0.75 & $0.56-0.99$ \\
\hline 23vPPV & 80 & 76.2 & 21.3 & 58 & 65.2 & 13.6 & 0.64 & $0.46-0.90$ \\
\hline $7 \mathrm{vPCV}$ & 60 & 57.1 & 16.0 & 15 & 16.9 & 3.5 & 0.22 & $0.12-0.39$ \\
\hline 23vPPV only & 20 & 19.0 & 5.3 & 43 & 48.3 & 10.1 & 1.90 & $1.12-3.22$ \\
\hline Non-7vPCV & 28 & 26.7 & 7.5 & 67 & 75.3 & 15.7 & 2.11 & $1.36-3.28$ \\
\hline Non-vaccine & 8 & 7.6 & 2.1 & 24 & 27.0 & 5.6 & 2.65 & $1.19-5.90$ \\
\hline $6 v(13 v-7 v)$ & 12 & 11.4 & 3.2 & 33 & 37.6 & 7.8 & 2.43 & $1.25-4.70$ \\
\hline
\end{tabular}

Note: $\quad 23 v P P V$ only = 23vPPV - 7vPCV; non-7vPCV = total $-(7 v P C V+$ untyped $)$; and non-vaccine $=$ total $-(23 P P V+$ untyped).

\section{DISCUSSION}

This investigation supports other reports that show the decline in the notification rate of IPD in children can be directly attributed to the use of 7vPCV. ${ }^{6}$ The decline in notification rates in adults is most likely an indirect effect of 7vPCV programme in children. ${ }^{7}$ This is supported by the recorded increase in the notification rate of IPD caused by 23vPPV-only types across most age groups. A similar effect has been described in another regional area of Australia ${ }^{4}$ and internationally. ${ }^{7}$

The increase in incidence of 23vPPV-only types across all age groups and less than 
$60 \%$ immunization coverage in the greater than 65 years age group suggests that 23vPPV has provided individual protection rather than a population effect. ${ }^{8}$ Increased nasopharyngeal carriage of 23vPPV-only types in vaccinated children may have had a negative effect on the impact of 23vPPV. ${ }^{4}$ Varied immunogenicity in the greater than 65 years age group due to comorbidities may have also had a negative impact on the efficacy of 23PPV. ${ }^{9}$

The increase in non-vaccine serotypes across all age groups is consistent with the phenomenon of serotype replacement that has been described elsewhere. ${ }^{10}$ Serotype 19A has emerged as a potent disease-causing organism, with its capsular switching and increasing antibiotic resistance, it has been able to evade vaccine selection pressure. $^{11}$ Serotype $19 \mathrm{~A}$ is one of the serotypes contained in the newly introduced $13 \mathrm{vPCV}$. If the distribution of serotypes remains the same, there is potential for a further $21 \%$ decline in the incidence of IPD caused by 19A in adults in the study population through a population effect.

The 13vPCV also presents an opportunity to further reduce the burden of IPD in children. In the zero to four year age group, $40.6 \%$ of all notified IPD cases during the 2008-2010 period were caused by $6 \mathrm{v}(13 \mathrm{v}-7 \mathrm{v})$ types. This result is similar to the findings of a national study in $2007 .^{3}$

IPD rates in males were observed to be higher than that of females. This phenomenon was observed across all age groups in both comparison periods and has been documented elsewhere. ${ }^{12}$ Continued surveillance of IPD is essential in monitoring the impacts of the pneumococcal vaccines and trends in serotypes.

There are limitations to this study. The decline in $6 v(13 v-7 v)$ types in the 5-14 years age group differs to trends in other age groups and may represent lack of precision with relatively small numbers. However, our findings of a population effect due to the $7 \mathrm{vPCV}$ are consistent with findings from another regional area of Australia, in North Queensland. 3,4

The 35\% decline in IPD notification rates in the 15-64 year age group is markedly different to the $2.6 \%$ decline found in North Queensland. This may be explained by the difference in study periods. The study period 2008-2010 is later than the 2006-2009 study period in north Queensland, which may have given 7vPCV more time to impact the population. Regional differences in serotypes and immunization rates may also have an impact and limit the ability of this study to be extrapolated to other regional areas of Australia. ${ }^{10}$ Pneumococcal vaccine coverage in the Hunter New England Local Health District before the introduction of the funded programmes in 2005 is unknown. Over $95 \%$ of children aged $24-27$ months in HNE were immunized in the 2008-2010 study period. ${ }^{13}$ High immunization coverage are associated with increases in non-vaccine types. ${ }^{10}$

No changes were made to surveillance or to IPD treatment protocols during the study periods. This study is also limited by the quality of the current surveillance system where several factors affect the notifiable fraction for communicable diseases in rural and regional areas in Australia, such as decreased seeking of medical care and decreased collection and submission of specimens. ${ }^{14}$ No outbreaks of IPD were recorded during this time.

\section{CONCLUSION}

The 7vPCV has proven to be an effective health intervention in reducing the pneumococcal disease burden in a regional area of Australia despite some level of serotype replacement. There is a potential for continuous serotype replacement after the introduction of $13 \mathrm{vPCV}$ as has happened with 7vPCV, but as with $7 \mathrm{vPCV}$, the gains made with $13 \mathrm{vPCV}$ are likely to improve health outcomes for both children and adults. The increase in cases due to serotypes in 13vPCV implies that $13 v P C V$ presents an opportunity to further reduce the burden of IPD in the Hunter New England Local Health District.

\section{Conflicts of interest}

None declared.

\section{Funding}

This work was supported by HNE Population Health.

\section{Acknowledgements}

The authors would like to acknowledge Maggi Osbourn, Clinical Nurse Consultant, for assistance in developing 
the project concept and data interpretation. The authors would also like to acknowledge Professor David Durrheim, Director of Health Protection, Hunter New England Population Health, for his comments on the manuscript.

\section{References:}

1. National Health and Medical Research Council. The Australian Immunisation Handbook 2008. 9th Edition. Canberra, Australian Government, 2008.

2. Mclntyre PB et al. Epidemiology of invasive pneumococcal disease in urban New South Wales, 1997-1999. The Medical Journal of Australia, 2000, 173 Suppl:S22-26. pmid:11062802

3. Williams $\mathrm{S}$ et al. Changing epidemiology of invasive pneumococcal disease in Australian children after introduction of a 7 valent pneumococcal conjugate vaccine. The Medical Journal of Australia, 2010, 194(3): 392-396. pmid:21299484

4. Hanna JN et al. Invasive pneumococcal disease in non-Indigenous people in north Queensland, 2001-2009. The Medical Journal of Australia, 2010, 193:392-396. pmid:20919968

5. Hunter New England Population Health. HEALTHeRESOURCE. New South Wales, Hunter New England Health, 2010 (http://www2. hnehealth.nsw.gov.au/HNEPH/HHNE/dem/demHNEpoppyr.htm, accessed 20 February 2012).

6. World Health Organization. Changing epidemiology of pneumococcal serotypes after introduction of conjugate vaccine: July 2010 report. Weekly Epidemiological Record, 2010, 83(43):435-436 (http://www.who.int/wer/2010/wer8543.pdf, accessed 7 May 2012).

7. Miller $E$ et al. Herd immunity and serotype replacement 4 years after seven-valent pneumococcal conjugate vaccination in
England and Wales: an observational cohort study. The Lancet Infectious Diseases, 2011, 11:760-768. doi:10.1016/S14733099(11)70090-1 pmid:21621466

8. New South Wales Population Health Survey. 2009 Summary Report on Adult Health. Vaccinated against pneumococcal disease in the last 5 years by area health service in NSW. North Sydney, New South Wales Department of Health, 2010 ( http://www.health. nsw.gov.au/resources/publichealth/surveys/hsa_09summary.pdf, accessed 20 October 2011).

9. Ridda I et al. Immunological responses to pneumococcal vaccine in frail older people. Vaccine, 2009, 27:1628-1636. doi:10.1016/j. vaccine.2008.11.098 pmid:19100304

10. Hanquet $\mathrm{G}$ et al. Pneumococcal serotypes in children in 4 European countries. Emerging Infectious Diseases, 2010, 16:1428-1439. doi:10.3201/eid1609.100102 pmid:20735928

11. Bruggeman A et al. Vaccine escape recombinants emerge after pneumococcal vaccination in the United States. PLoS Pathogens, 2007, 3:1628-1635. doi:10.1371/journal.ppat.0030168 pmid: 18020702

12. Annual Epidemiological Report on Communicable Diseases in 2009. Stockholm, European Centre for Disease Prevention and Control, 2009, 166-168 (http://ecdc.europa.eu/en/ publications/Publications/0910_SUR_Annual_Epidemiological_ Report_on_Communicable_Diseases_in_Europe.pdf, accessed 7 May $\overline{20} \overline{1} 2$ ).

13. Australian Childhood Immunisation Register Coverage Report. LGA by age group, 2008-2010. Canberra, Australian Government Department of Human Services, 2011.

14. Slaon-Gardner T et al.; NNDSS Annual Report Writing Group. Australia's notifiable disease status, 2009: annual report of the National Notifiable Diseases Surveillance System. Communicable Diseases Intelligence, 2011, 35:61-131. pmid:22010505 